\title{
Dynamics of Coupled Self-Pulsating Semiconductor Lasers
}

\author{
Alessandro Scirè, Member, IEEE, Claudio Juan Tessone, and Pere Colet
}

\begin{abstract}
We introduce and analyze a model for the dynamics of two coupled self-pulsating semiconductor lasers. We investigate the role of the complex coupling coefficient in the static and dynamic properties of the device. We find conditions for the emergence of coherent laser pulses, in which the two lasers display synchronous coherent self-pulsations (self-pulsating super modes). Nonlinear dynamics and two different routes to chaos are also individuated and discussed.
\end{abstract}

Index Terms-Chaos, laser array, laser dynamics, self-pulsations, semiconductor lasers.

\section{INTRODUCTION}

$\mathbf{I}$ NTRACAVITY saturable absorption is a well-established method to achieve pulsating output from a laser source. It is used both in mode-locking and $Q$-switching techniques to produce such behavior. In the mode-locking (ML) technique [1], several spectral optical modes cooperatively act to build-up a laser pulse, once the stable continuous-wave (CW) operation is inhibited by a saturable absorber. The $Q$-switching technique can profit from the presence of a saturable absorber as well: the pumping excites population inversion above the threshold value, producing an increase in field intensity as soon as the unsaturated gain overcomes the losses. The large increase in intensity saturates the absorber, which in turn leads to large output powers and to the saturation of the gain in the active zone. Owing to the gain depletion, the intensity decays to the noise level and the population inversion in the absorber decreases. The self-sustained repetition of this process yields a regular train of pulses. Spontaneous self $Q$-switching in semiconductor lasers was recognized in narrow proton-stripe edge-emitting semiconductor lasers (EELs) [2], [3], due to the saturable absorption of the unpumped region surrounding the pumped waveguide. Since those lasers were self-pulsating, this phenomenon was called self-pulsations (SP). SPs are intimately related to $Q$-switching; indeed, SPs coincide to what is called passive $Q$-switching [4].

Laser pulses are useful in several applications, from optical communications to nonlinear optics. In this view, SPs in semiconductor lasers were intensively investigated [5]-[8] and have found commercial applications in optical-disk systems due to their low sensitivity to optical feedback ([9, p. 190]); also, SPs

\footnotetext{
Manuscript received August 23, 2004; revised October 13, 2004. This work was supported by the European Commission under VISTA HPRN-CT-200000034, under the OCCULT Project 2000- 29683, and by the Spanish MCyT under Project BFM2000-1108, MCyT and Feder SINFIBIO BFM 2001-0341$\mathrm{C} 02-02$.

The authors are with the Instituto Mediterráneo de Estudios Avanzados (IMEDEA), Consejo Superior de Investigaciones Científicas, Universitat de les Illes Balears, E-07122 Palma de Mallorca, Spain (e-mail: scire@ imedea.uib.es). Digital Object Identifier 10.1109/JQE.2004.841929
}

were proposed for further applications in clock extraction systems [10] and in synchronization schemes for encoded communication [11], [12].

However, SPs in a single semiconductor laser are limited in power to a few milliwatts and often display fluctuations and instabilities that frustrate their potential applications, e.g., in optical communications. A substantial improvement could come from the achievement of synchronized (coherent) pulsed output of many coupled laser sources. This study aims to improve the understanding of pulsating behavior in coupled lasers in view of the generation of high-power coherent laser pulses from semiconductor laser arrays.

In this paper, we introduce a model to describe the temporal dynamics of the electric field and carrier populations in two EELs-each given an unpumped saturable absorption section-and laterally coupled through evanescent wave. The structure resembles the well-known Twin-Stripe [13], but here each stripe has two sections, an active one and an absorbing one. The interelement field dynamics is the new feature with respect to the well-known self-pulsating EELs. Our description of the absorbing region relies on the standard one developed for the two-section EEL [14]. The interelement optical coupling is modeled as in [15]. Combining the two approaches, we are able to investigate the dynamics of a laterally two-coupled semiconductor laser, each stripe including a saturable absorber. The case of two-coupled lasers is of particular interest since it allows a complete analytical study and provides a good physical insight in view of a more general study of a many-element array of pulsating lasers, because all of the coherent behaviors (e.g., synchronized pulses) are likely to be present in the many-element case as well.

This paper is organized as follows. In Section II, we present a detailed description of the model. In Section III, we show the results of the linear stability analysis and we characterize the different dynamical regimes. In Section IV, we describe the the routes to optical chaos. Finally, in Section V, we summarize this paper.

\section{MODEL}

We consider a laser structure consisting of two adjacent EELs, schematically depicted in Fig. 1. Each EEL has a first pumped section providing gain (labeled $p$ ) and a second region (labeled $a$ ) acting as a saturable absorber. Physically, the two lasers are optically coupled due to diffraction, whereas in each laser the pumped and absorbing regions are coupled to each other by carrier diffusion. We neglect further sources of coupling such as thermal ones and cross-carrier diffusion between lasers 1 and 2 . 
a) Top view

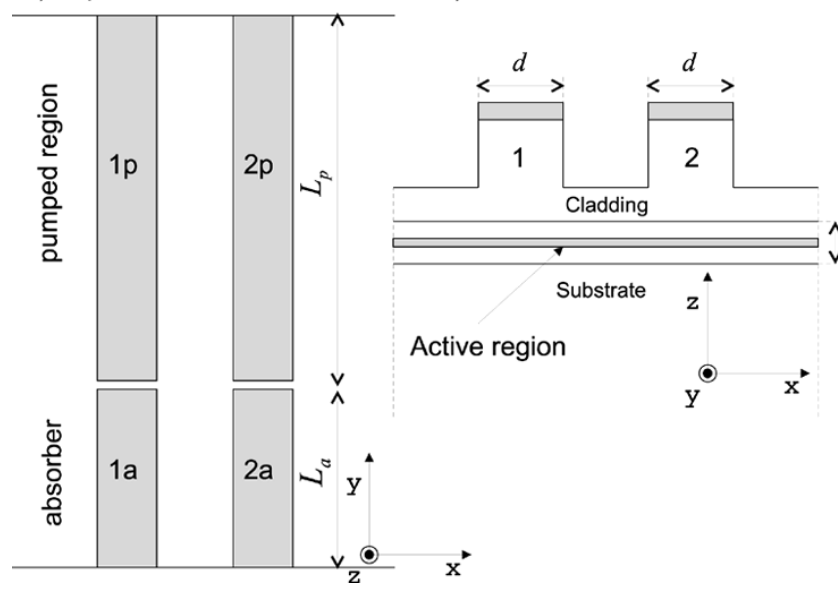

Fig. 1. Schematic picture of the device.

We assume that each unperturbed guide supports a single longitudinal mode, and thus the total transverse electric field is written as a linear superposition of the unperturbed individual waveguide fields and the residual radiation field is neglected [14]. The analysis of diffraction-induced crosstalk in terms of the coupling between modes of individual waveguides is formally exact [16], but the ability to analyze such a problem is restricted to weakly guiding structures which are sufficiently well separated. The result of such analysis consists of a linear complex coupling term between the two fields, which is quantitatively individuated by the waveguide parameters (mainly the distance between the waveguides). Indeed, the actual values of the coupling parameters stem from the eigenvalue analysis of the coupled waveguides and, in general, are found to be technologically (or even device-) dependent. In our approach, we leave those coupling terms as free parameters of our model. The spatial wave propagation problem is therefore simplified in to rate equations for the modal amplitudes. The equations governing the optical and material variables read

$$
\begin{aligned}
\frac{d E_{i}\left(t^{\prime}\right)}{d t^{\prime}}= & \frac{1+j \alpha}{2}\left[g_{p} \xi_{p}\left(N_{p i}-N_{t p}\right)+g_{a} \xi_{a}\left(N_{a i}-N_{t a}\right)-\kappa\right] \\
& \cdot E_{i}-\left(k_{d}+j k_{c}\right) E_{m}+\mathcal{G}_{i} \\
\frac{d N_{p i}\left(t^{\prime}\right)}{d t^{\prime}}= & \frac{J_{i}}{e V_{p}}-\gamma_{e p} N_{p i}-\frac{N_{p i}-N_{a i}}{T_{p a}} \\
& -\frac{g_{p} \xi_{p}}{V_{p}}\left(N_{p i}-N_{t p}\right)\left|E_{i}\right|^{2} \\
\frac{d N_{a i}\left(t^{\prime}\right)}{d t^{\prime}}= & -\gamma_{e a} N_{a i}-\frac{\left(N_{a i}-N_{p i}\right)}{T_{a p}}-\frac{g_{a} \xi_{a}}{V_{a}}\left(N_{a i}-N_{t a}\right)\left|E_{i}\right|^{2}
\end{aligned}
$$

where $i=1,2, m=3-i, j$ is the imaginary unit, $E_{i}$ is the slowly varying complex amplitude of the electric field of the optical mode supported by waveguide $i$, and $N_{p i}\left(N_{a i}\right)$ is the carrier inversion density between the conduction and valence bands in the pumped (absorbing) regions of laser $i$. The meaning and typical values of the different parameters in the model (1)-(3) are given in Table I; however, two important points are worth mentioning. The dependence of the gain with the carrier density has been substituted by a two-linear approximation by taking different values of $g_{p, a}, N_{t p}$, and $N_{t a}$ depending on whether the region $p$ or $a$ is considered. Second, we define a characteristic volume of the regions $V_{p} \equiv w d L_{p}$ and $V_{a} \equiv w d L_{a}$ with $w$ denoting the quantum-well (QW) thickness, $d$ is the waveguide thickness, and $L_{p}$ and $L_{a}$ are the lengths of the pumped regions and absorbers, respectively. The parameter $\xi_{p, a}$ represents the fraction of the intensity of the electric field that lays in regions $p$ and $a$ [6] and whose values are given by the integrals of the spatial mode profile over regions $p$ and $a$. By choosing a standard expression for the spatial profile in the single longitudinal and transverse-mode operation conditions (e.g., see [9, p. 232]), one finds that $\xi_{p} / \xi_{a} \approx L_{p} / L_{a}$, under the approximation that $L_{p, a} \gg \lambda$, where $\lambda$ is the laser wavelength. Normalization conditions impose $\xi_{p}+\xi_{a}=1$. The two diffusion times vary according to the volumes of the regions $V_{p} / V_{a} \approx$ $T_{p a} / T_{a p}$ [5], [6]. The linewidth enhancement factor $\alpha$ describes the phase-amplitude coupling mechanisms. As previously discussed, the coupling between the two self-pulsating lasers is described in terms of the parameters $k_{d}$ and $k_{c}$. The dissipative coupling term $k_{d}$ represents additional optical losses in the region between the two lasers, where the two field modes interfere. The conservative (or coherent) coupling of the two localized field modes by optical diffraction is represented by $k_{c}$. Finally, spontaneous emission processes are accounted through two independent Langevin noise sources $\mathcal{G}_{1,2}(t)$. We remark that, in the model (1)-(3), the two lasers are individually described by the simplest model for a semiconductor laser including saturable absorptive effects: rate equations based on individual single-mode oscillations [17]. Moreover, for the sake of completeness, a rigorous derivation of our model (1)-(3) would rely on a consistent treatment of diffusion and diffraction effects [18]. The main approximations that make our model consistent with the full spatial approach are the same as discussed in [19] and [20].

\section{A. Dimensionless Model}

For the sake of simplicity and numerical purposes, we rescale the dynamical variables by

$$
\begin{aligned}
F & =\sqrt{\frac{g_{p}}{V_{p}} \frac{\xi_{p}}{\gamma_{p} \kappa}} E \\
D_{p} & =\frac{g_{p} \xi_{p}}{\kappa}\left(N_{p}-N_{t p}\right) \\
D_{a} & =\frac{g_{a} \xi_{a}}{\kappa}\left(N_{a}-N_{t a}\right) .
\end{aligned}
$$

With these new variables, (1)-(3) reduce to

$$
\dot{F}_{i}(t)=\frac{1+j \alpha}{2}\left[D_{p i}+D_{a i}-1\right] F_{i}-\left(\varepsilon_{d}+j \varepsilon_{c}\right) F_{m}+f_{i}(t)
$$

$\dot{D}_{p i}(t)=\gamma_{p}\left[A_{p i}-D_{p i}\left(1+\left|F_{i}\right|^{2}\right)+c_{p a} D_{a i}\right]$

$\dot{D}_{a i}(t)=\gamma_{a}\left[A_{a i}-D_{a i}\left(1+r\left|F_{i}\right|^{2}\right)+c_{a p} D_{p i}\right]$

where

$$
\gamma_{a, p} \equiv \frac{1}{\kappa}\left(\gamma_{e a, p}+\frac{1}{T_{a p, p a}}\right)
$$


TABLE I

MEANING AND TYPICAL VALUES OF THE PARAMETERS

\begin{tabular}{|c|c|c|c|}
\hline Symbol & Meaning & Value & Dimensions \\
\hline $\bar{\alpha}$ & linewidth enhancement factor & 3 & - \\
\hline$g_{p}$ & gain coefficient pumped region $p$ & $2 \times 10^{-16}$ & $\mathrm{~cm}^{3} \mathrm{~ns}^{-1}$ \\
\hline$g_{a}$ & gain coefficient absorbing region $a$ & $2.8 \times 10^{-16}$ & $\mathrm{~cm}^{3} \mathrm{~ns}^{-1}$ \\
\hline$\xi_{p}$ & field fraction in region $p$ & 0.37 & - \\
\hline$\xi_{a}$ & field fraction in region $a$ & 0.63 & - \\
\hline$N_{t p}$ & transparency carrier density pumped region $p$ & $10^{18}$ & $\mathrm{~cm}^{-3}$ \\
\hline$N_{t a}$ & transparency carrier density absorbing region $a$ & $2 \times 10^{18}$ & $\mathrm{~cm}^{-3}$ \\
\hline$V_{p}$ & pumped region volume & $w d L_{p}$ & $\mathrm{~cm}^{3}$ \\
\hline$V_{a}$ & absorbing region volume & $w d L_{a}$ & $\mathrm{~cm}^{3}$ \\
\hline$J_{1(2)}$ & current supplied in the pumped region $p$ of laser 1 (2) & & $m A$ \\
\hline$L_{p} / L_{a}$ & pumped/absorbing region length ratio & 1.65 & - \\
\hline$L_{p}$ & pumped region length & 200 & $\mu m$ \\
\hline$d$ & waveguide thickness & 2 & $\mu m$ \\
\hline$w$ & active region thickness & 0.45 & $\mu m$ \\
\hline$T_{p a}$ & diffusion time from region $p$ to region $a$ & 2 & ns \\
\hline$\kappa$ & cavity decay rate (facet reflectivity $=0.3$ ) & 100 & $\mathrm{~ns}^{-1}$ \\
\hline$\gamma_{e p}$ & spontaneous decay rate in the pumped region & 0.95 & $\mathrm{~ns}^{-1}$ \\
\hline $\begin{array}{c}\gamma_{e a} \\
\beta\end{array}$ & $\begin{array}{l}\text { spontaneous decay rate in the absorbing region } \\
\text { spontaneous emission factor }\end{array}$ & $\begin{array}{l}2 \times \gamma_{e p} \\
10^{-5}\end{array}$ & $\mathrm{~ns}^{-1}$ \\
\hline
\end{tabular}

$$
\begin{aligned}
c_{a p} & \equiv \frac{g_{a} \xi_{a}}{g_{p} \xi_{p}} \frac{1}{\gamma_{a} \kappa T_{a p}} \\
c_{p a} & \equiv \frac{g_{p} \xi_{p}}{g_{a} \xi_{a}} \frac{1}{\gamma_{p} \kappa T_{p a}} \\
A_{p} & \equiv \frac{g_{p} N_{t p} \xi_{p}}{\kappa}\left[\frac{J}{J_{t}}-1+\frac{N_{t a}}{N_{t p}} \frac{1}{\kappa \gamma_{p} T_{a p}}\right] \\
A_{a} & \equiv \frac{g_{a} N_{t a} \xi_{a}}{\kappa}\left[-1+\frac{N_{t p}}{N_{t a}} \frac{1}{\kappa \gamma_{a} T_{p a}}\right] \\
r & \equiv \frac{g_{a} \xi_{a}}{g_{p} \xi_{p}} \frac{\gamma_{p}}{\gamma_{a}} \\
\varepsilon_{d} & \equiv \frac{k_{d}}{\kappa} \\
\varepsilon_{c} & \equiv \frac{k_{c}}{\kappa} .
\end{aligned}
$$

We have defined the transparency current as $J_{t} \equiv e \kappa \gamma_{p} N_{t p} V_{p}$. The rescaling is the same for lasers 1 and 2 , so we have dropped that index in (4)-(6), (12), and (13). Equations (7)-(9) are written in a dimensionless form such that the dimensionless time $t^{\prime}=\kappa t$. The dot acting on the dynamical variables means derivative with respect to $t$. The rescaled dissipative $\left(\varepsilon_{d}\right)$ and conservative $\left(\varepsilon_{c}\right)$ are now the coupling parameters. The effective injection currents, with respect to the transparency value, are $A_{p}$ and $A_{a}$. Carrier diffusion is present in the equations through the coupling terms $c_{p a}$ and $c_{a p}$.

The Langevin noise sources $f_{i}(t)$ can be approximated by

$$
f_{i}(t)=\sqrt{\beta \xi_{p} \gamma_{p} D_{p i}+\beta \xi_{a} \gamma_{a} D_{a i}} \zeta_{i}(t)
$$

where $\beta \xi_{a, p}$ represents the fraction of the spontaneously emitted photons that goes into the zone $a$ or $p$ of the lasing mode, and $\zeta_{1,2}$ are two independent complex Gaussian random numbers, with zero mean $\left\langle\zeta_{i}(t)\right\rangle=0$ and correlation $\left\langle\zeta_{i}(t) \zeta_{j}^{*}\left(t^{\prime}\right)\right\rangle=$ $2 \delta_{i j} \delta\left(t-t^{\prime}\right)$.

\section{B. Stationary Solutions}

In the following, we assume symmetric operation conditions $A_{p i}=A_{p}$ and $A_{a i}=A_{a}$. The electric field solution of (7)-(9) is expressed as $F_{i}(t)=Q_{i} e^{j\left(\omega_{i} t \pm \varphi\right)}$. We start our discussion by looking at the symmetric stationary solutions (SSs), i.e., $Q_{i}=$ $Q, \omega_{i}=\omega$, and $\dot{D}_{p i}=\dot{D}_{a i}=0$. We find two types of SSs (resembling the super-modes in the twin-stripe [15] structure): $\varphi=0$, in-phase (IP), and $\varphi=\pi / 2$, out-of-phase (OP). The emission frequency (in $\kappa$ units) is $\omega= \pm\left(\varepsilon_{d} \alpha-\varepsilon_{c}\right)$, where the sign $+(-)$ stands for an IP (OP) solution [labeled as IP (OP) supermodes in the following]. By defining $I=Q^{2}$, the stationary carrier inversions are in turn given by

$$
\begin{aligned}
\bar{D}_{p i} & =\frac{A_{p}(1+r I)+A_{a} c_{p a}}{(1+I)(1+r I)-c_{p a} c_{a p}} \\
\bar{D}_{a i} & =\frac{A_{a}(1+I)+A_{p} c_{a p}}{(1+I)(1+r I)-c_{p a} c_{a p}}
\end{aligned}
$$

by dropping for simplicity the index $i$, and $\bar{D}_{p}$ and $\bar{D}_{a}$ satisfy gain clamping condition $\bar{D}_{p}+\bar{D}_{a}=\pi_{d}$, where $\pi_{d} \equiv 1 \pm 2 \varepsilon_{d}$ and the sign $+(-)$ stands for an IP (OP) solution. This leads to a quadratic equation for $I$ that reads

$$
\begin{aligned}
r \pi_{d} I^{2}+\left[(1+r) \pi_{d}\right. & \left.-r A_{p}-A_{a}\right] I+\pi_{d}\left(1-c_{p a} c_{a p}\right) \\
& -A_{p}\left(1+c_{a p}\right)-A_{a}\left(1+c_{p a}\right)=0 .
\end{aligned}
$$

The first laser threshold is defined as the pump value for which the off-solution $I=0$ loses stability. By taking $I=0$ in (17)-(18) and imposing the gain clamping condition, we find that the threshold currents for the two solutions OP and IP are given by

$$
A_{p \mathrm{Th}}^{(I P, O P)}=\frac{\left(1-c_{p a} c_{a p}\right) \pi_{d}^{(I P, O P)}-A_{a}\left(1+c_{p a}\right)}{1+c_{a p}} .
$$

Equation (20) can be interpreted as follows. There is an increase in threshold current due to the absorption $\left(A_{a}<0\right)$ and due to the carrier diffusion from region 1 to region $2\left(c_{p a}\right)$, while the threshold decreases if the inverse carrier flux is favored $\left(c_{a p}\right)$. Either for $\pi_{d}^{(O P)}$ or $\pi_{d}^{(I P)}$, (19) has only one physical solution (positive root) for $A_{p}>A_{p} \mathrm{Th}$. For sufficiently intense absorbing conditions, three solutions appear in a narrow range 
of currents $A_{p} \lesssim A_{p \text { Th }}$ producing a hysteresis cycle [8], [17]: stable solutions with $I=0$, high-power solutions, and intermediate-power solutions that result in unstable states.

\section{LINEAR ANALYSIS}

A linear stability analysis of the two SSs of the system (7)-(9) can be carried out by introducing a small perturbation, yielding

$$
\begin{aligned}
F_{i}(t) & =\left(Q+\eta_{i}(t)\right) e^{j(\omega t \pm \varphi)} \\
D_{p i}(t) & =\bar{D}_{p}+\Delta_{p i}(t) \\
D_{a i}(t) & =\bar{D}_{a}+\Delta_{a i}(t)
\end{aligned}
$$

where $\eta_{i}$ is a complex perturbation of the field amplitude, and $\Delta_{p i}$ and $\Delta_{a i}$ are real-value perturbations of the carrier variables. Upon substituting (21)-(23) into the equations of the model (7)-(9) and linearizing, we obtain a set of coupled linear differential equations for the perturbations which, written for the variables $S \equiv \eta_{1}+\eta_{2}, T_{p}=\Delta_{p 1}+\Delta_{p 2}, T_{a}=\Delta_{a 1}+\Delta_{a 2}$ and $R \equiv \eta_{1}-\eta_{2}, \tau_{p}=\Delta_{p 1}-\Delta_{p 2}, \tau_{a}=\Delta_{a 1}-\Delta_{a 2}$ decouples into two subsets. The first subset $\left(S, T_{p}, T_{a}\right)$ determines the stability of the intensities of the two supermodes, whereas the second subset $\left(R, \tau_{p}, \tau_{a}\right)$ describes the stability of the relative phase, as discussed below.

\section{A. Intensity Stability of the Supermodes and Self-Pulsation Conditions}

The actual dimension of the subset $\left(S, T_{p}, T_{a}\right)$ is $3 \times 3$, due to the presence of an invariant global phase, that implies the constant presence of a zero eigenvalue. Therefore, by introducing $P=S+S^{*}$, we have

$$
\begin{aligned}
\dot{P}(t) & =Q\left(T_{p}+T_{a}\right) \\
\dot{T}_{p}(t) & =\gamma_{p}\left[-Q \bar{D}_{p} P-\left(1+Q^{2}\right) T_{p}+c_{p a} T_{a}\right] \\
\dot{T}_{a}(t) & =\gamma_{a}\left[-r Q \bar{D}_{a} P-\left(1+r Q^{2}\right) T_{a}+c_{a p} T_{p}\right]
\end{aligned}
$$

which determines the stability of the intensities (see the discussion below) of each supermode. The characteristic equation for the eigenvalues $s$ of the linearized system (24)-(26) obey a third-order polynomial

$$
s^{3}+C_{2} s^{2}+C_{1} s+C_{0}=0 .
$$

In this expression,

$$
\begin{aligned}
C_{0}= & \gamma_{p} \gamma_{a} I\left[\bar{D}_{p}\left(1+r I+c_{a p}\right)+\bar{D}_{a}\left(1+I+c_{p a}\right)\right] \\
C_{1}= & \gamma_{p} \gamma_{a}(1+I)(1+r I)-\gamma_{p} \gamma_{a} c_{p a} c_{a p}+\gamma_{p} I \bar{D}_{p} \\
& +\gamma_{a} r I \bar{D}_{a} \\
C_{2}= & \gamma_{a}(1+r I)+\gamma_{p}(1+I) .
\end{aligned}
$$

By applying the Routh-Hurwitz criterion, when the condition $C_{2} C_{1}=C_{0}$ is fulfilled, the total intensity loses stability through a Hopf bifurcation, giving rise to intensity pulsations at frequency $\Omega=\sqrt{C_{1}}$. By taking advantage of the small values of $\gamma_{p}$ and $\gamma_{a}$, as in [21], the condition $C_{2} C_{1}=C_{0}$ is well approximated by

$$
(1+I) \bar{D}_{p}+\frac{\gamma_{a}^{2}}{\gamma_{p}^{2}}(1+r I) \bar{D}_{a}-\frac{\gamma_{a}}{\gamma_{p}}\left(r \bar{D}_{a} c_{p a}+\bar{D}_{p} c_{a p}\right)=0 .
$$

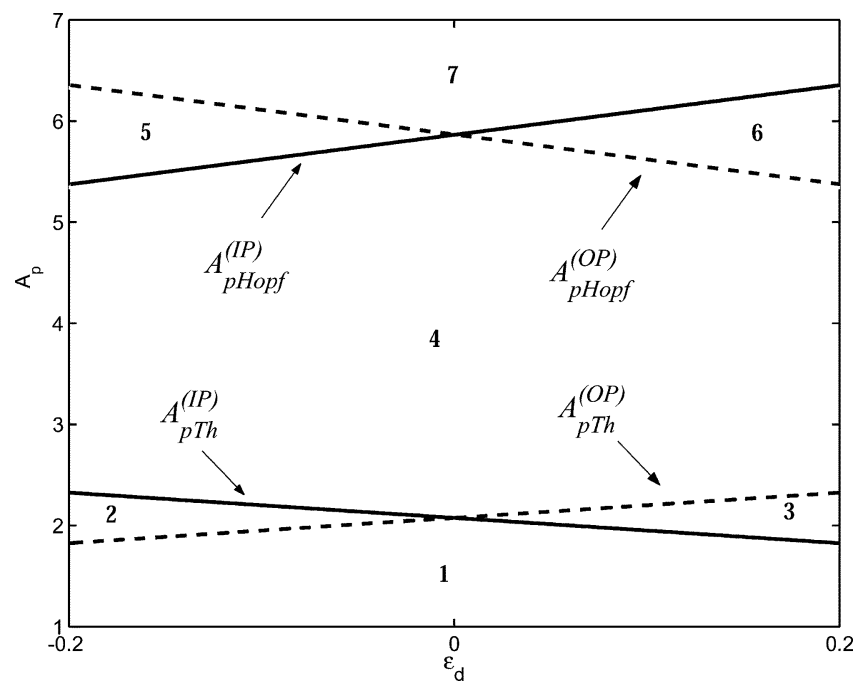

Fig. 2. Thresholds given by (20) and Hopf bifurcation boundaries given by (31) for IP (solid) and OP (dashed) supermodes.

Substituting the solutions for $\bar{D}_{a, p}$ reported in (17)-(18) and solving (19) for $I$ as function of $A_{p}$, (31) gives two values of $A_{p}\left(A_{p \text { Hopf }}^{(\mathrm{IP}, \mathrm{OP})}\right)$ that bound the Hopf-bifurcation locus for the IP and OP supermodes, respectively. Notice that the location of the Hopf bifurcation does not depend on the imaginary part of the coupling $\varepsilon_{c}$. It is also independent of the $\alpha$-factor. The study in [21] reported coefficients of the secular determinant describing the intensity linear stability in a single self-pulsating EEL, including nonlinear recombination effects. Considering only one laser, our coefficients (28)-(30) revert to those ones of [21] when nonlinear recombination effects are neglected. The subset (24)-(26) turns out to be very similar to the single self-pulsating laser case. The difference relies in the fact that our case presents two stationary symmetric solutions (IP and OP supermodes), and this is due to the coupling between the two sources.

The stability boundaries of the intensity subset (24)-(26) are shown in Fig. 2 as function of the dimensionless real part of the coupling and scaled pumping. The corresponding values for the real part of physical coupling $k_{d}$ can be calculated using (15) while the physical pumping is given by (12). In region 1 , both supermodes are below threshold, and there is no stimulated emission. In region 2, the OP supermode is above threshold while IP is below threshold. However, the OP intensity is unstable, therefore a pulsating emission of the OP supermode is expected. In region 3, the situation is reversed around and a pulsating IP emission is expected. In region $4 \mathrm{~s}$ both IP and OP supermodes are above threshold and allowed to pulsate. In region 5 (6), the OP (IP) supermode pulsates, whereas the IP (OP) supermode reaches a steady state ( $\mathrm{CW}$ operation). In region 7 , both supermodes reach a steady state. The self-pulsation frequency calculated above is shown in Fig. 3 for the two supermodes. The parameters are given in Table I.

We observe that the role of the dissipative coupling coefficient $\varepsilon_{d}$ is to give different losses to the two supermodes IP and $\mathrm{OP}$, therefore splitting the lines describing the oscillation onset and absorber saturation of each solution. The complete information about the supermode selection and stability is given by 

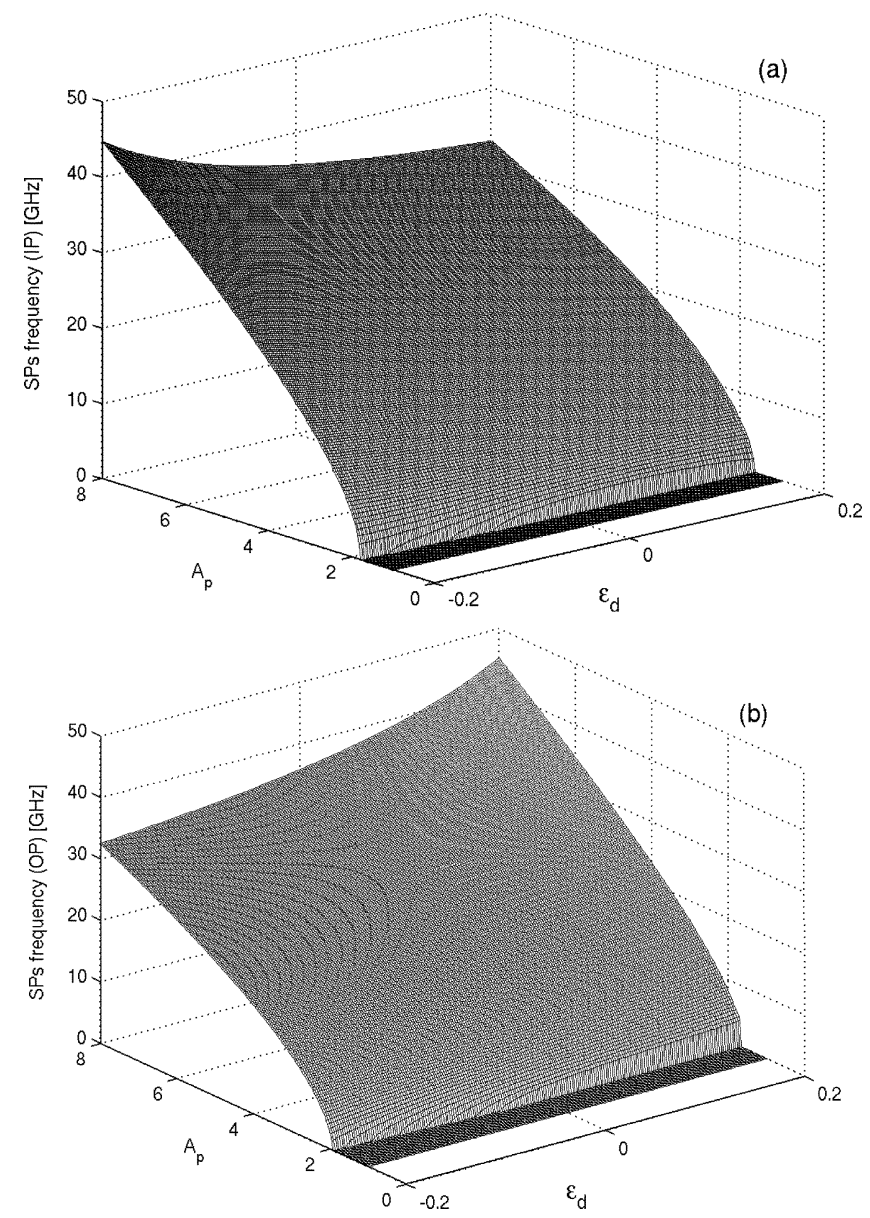

Fig. 3. Self-Pulsation frequency for the (a) IP and (b) OP supermodes.

the merging of the stability properties of the intensity and of the phase subset, which is the object of the next subsection.

\section{B. Phase Stability of the Supermodes}

The second linear subset represents the effect of a perturbation enhancing the difference between the dynamic variables of the two lasers, thus gives informations about the phase stability of the supermodes. It reads

$$
\begin{aligned}
\dot{R}(t) & =\frac{1}{2}(1+i \alpha) Q\left(\tau_{p}+\tau_{a}\right) \pm 2\left(\varepsilon_{d}+i \varepsilon_{c}\right) R \\
\dot{R}^{*}(t) & =\frac{1}{2}(1-i \alpha) Q\left(\tau_{p}+\tau_{a}\right) \pm 2\left(\varepsilon_{d}-i \varepsilon_{c}\right) R^{*} \\
\dot{\tau}_{p}(t) & =\gamma_{p}\left[c_{p a} \tau_{a}-Q \bar{D}_{p}\left(R+R^{*}\right)-\left(1+Q^{2}\right) \tau_{p}\right] \\
\dot{\tau}_{a}(t) & =\gamma_{a}\left[c_{a p} \tau_{p}-r Q \bar{D}_{a}\left(R+R^{*}\right)-\left(1+r Q^{2}\right) \tau_{a}\right]
\end{aligned}
$$

where $+(-)$ stands for the stability of the IP (OP) supermode. The phase stability depends explicitly on both the real and the imaginary part of the coupling as well as on the $\alpha$-factor. In fact it is the interplay between the coupling coefficients $\left(\varepsilon_{d, c}\right)$ and the $\alpha$-factor that determines the selection of the phase relationship between the two individual fields, and consequently the supermode selection. The subset (32)-(35) yields a fourth-order characteristic polynomial, that we analyze by numerical methods. We compute the phase stability boundaries for both IP and OP supermodes in the plane $A_{p}$ versus $\varepsilon_{c}$. We first consider the case $\varepsilon_{d}<0$, and $\varepsilon_{c}>0$. The OP supermode turns out to be always

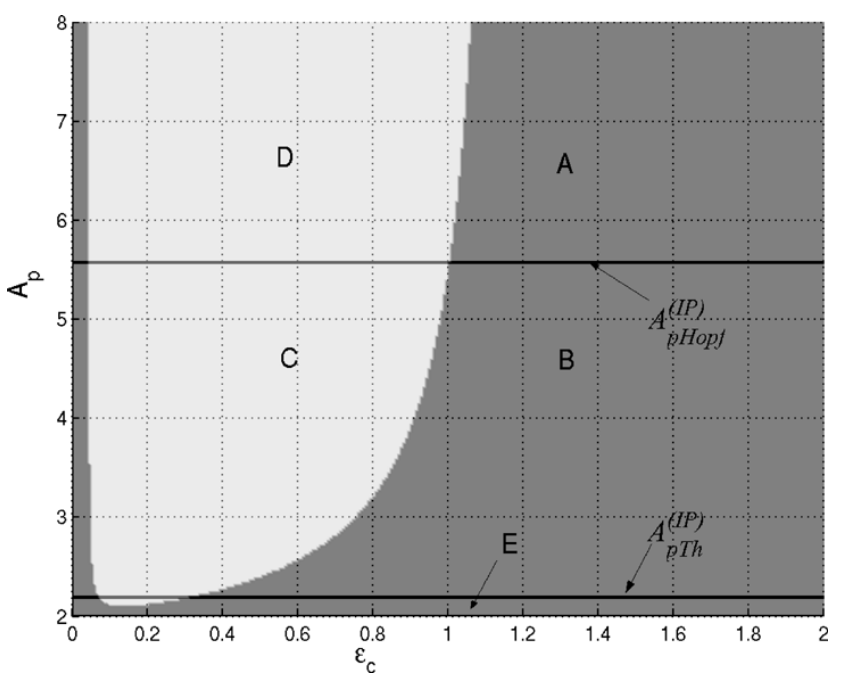

Fig. 4. Stability diagram in the plane $\varepsilon_{d}$ Vs $A_{p}$. (A) Stable in-phase output (stable IP supermode). (B) Pulsating in-phase output (pulsating IP supermode). (C) and (D) Unstable regions. (E) Below threshold. The diagram is relative to the IP solution. The black solid lines have the same meaning as in Fig. 2. The OP solution is always unstable for this parameter choice. $\varepsilon_{d}=-0.1$ and the remaining parameters are the same as in Table I .
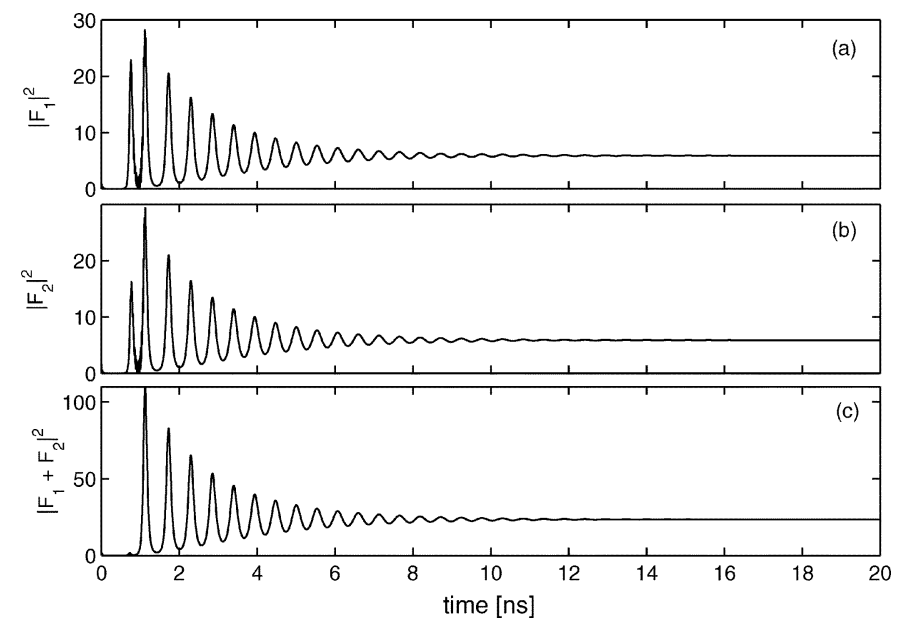

Fig. 5. Stable IP CW outputs (stable IP supermode). $A_{p}=6, \varepsilon_{c}=1.4$, and $\varepsilon_{d}=-0.1$.

unstable. Fig. 4 shows the stability diagram for the IP supermode in the $\left(A_{p}, \varepsilon_{c}\right)$ plane. There are four regions in which the dynamics is qualitatively different. In region $\mathrm{A}$, both subsystems (24)-(26) and (32)-(35) are stable. Therefore, the system shows stable CW-in-phase fields (stable IP supermode), the intensity of the electric field in each laser $\left|F_{i}\right|^{2}$ reaches a steady state (see Fig. 5), and the relative phase of the two fields goes to zero after a transient. The carriers reach the stationary value given by (17) and (18). In region B, the absorber is no longer saturated, the subsystem (24)-(26) is unstable, and pulsating output takes place. Formally, the self-pulsating solution arises as a consequence of a homoclinic bifurcation at threshold [5], which leads to the onset of a closed loop in the phase space [22], physically accounting for the field-medium energy exchange during the pulse. Increasing the pump current $A_{p}$, e.g., moving from region B toward region A of Fig. 4, the limit cycle shrinks and disappears through a Hopf bifurcation. As discussed in the previous subsection, the Hopf bifurcation locus does not depend 

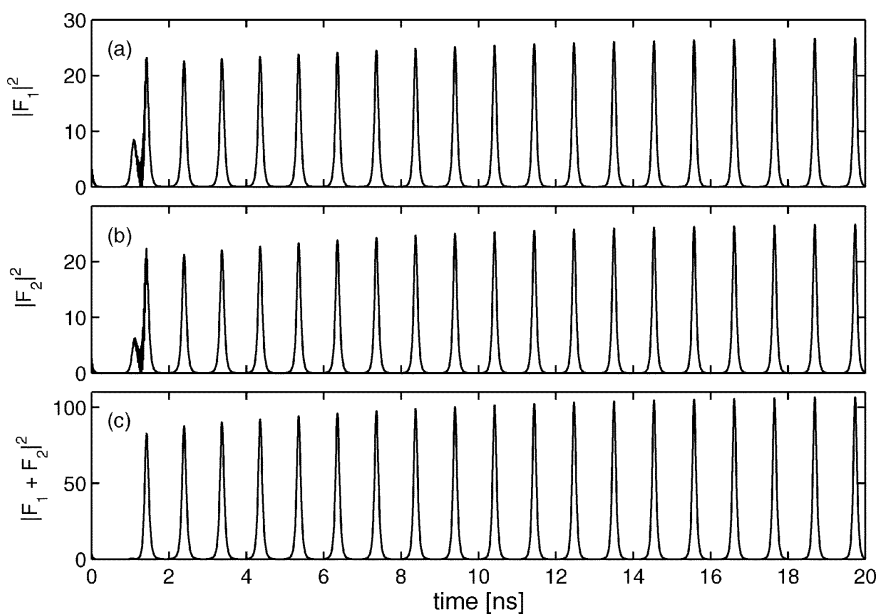

Fig. 6. IP pulsating outputs. Pulsating IP supermode. $A_{p}=3, \varepsilon_{c}=1.4$, and $\varepsilon_{d}=-0.1$.
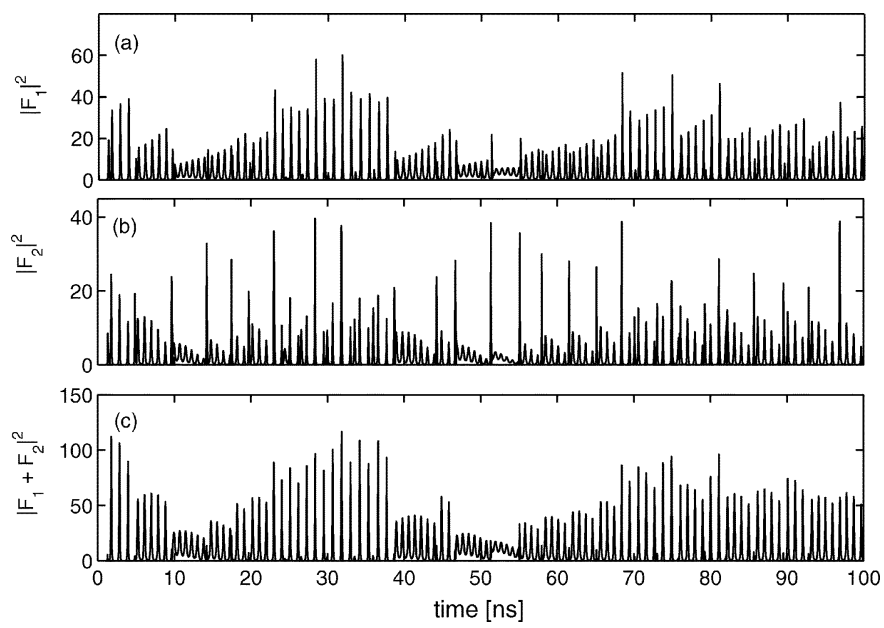

Fig. 7. Chaotic behavior with unsaturated absorber. $A_{p}=4, \varepsilon_{c}=0.4$, and $\varepsilon_{d}=-0.1$.

on $\varepsilon_{c}$ and is displayed as a horizontal solid line in Fig. 4. The subsystem (32)-(35) is stable, so that the intensity of the two lasers pulsates synchronously, namely, both lasers emit intensity pulses at the same time. Furthermore, both lasers emit coherently, with the same electric field phase. Fig. 6 shows the pulsating IP supermode: the intensity of the electric field in each laser $\left|F_{i}\right|^{2}$ reaches a stationary pulsating regime, and the relative phase of the two fields goes to zero. We remark that regimes in regions $\mathrm{A}$ and $\mathrm{B}$ are coherent regimes. Therefore, the intensity of the superposition of the two fields $\left|F_{1}+F_{2}\right|^{2}$ is four times the intensity of the single source.

In regions $\mathrm{C}$ and $\mathrm{D}$, the phase instability associated to the unstable eigenvalues of the subsystem (32)-(35) leads to the emergence of a complex nonlinear dynamics, in which chaotic behaviors take place (see Fig. 7). This is explained in Section IV. In region $\mathrm{E}$, the IP solution is below threshold $A_{p}<A_{p \text { Th }}^{(I P)}$ of (20), and the output intensity drops to zero.

Changing the precise value of $\varepsilon_{d}$ does not change qualitatively the stability diagram. For smaller values of $\left|\varepsilon_{d}\right|$, the instability regions widens, whereas it shrinks for larger values of $\left|\varepsilon_{d}\right|$.
For $\varepsilon_{d}>0$ and $\varepsilon_{c}<0$, the IP supermode is always unstable. The stability diagram of the OP supermode has different regions whose shape is the same as in Fig. 4 but changing $\varepsilon_{c} \rightarrow-\varepsilon_{c}$. In this case, region $\mathrm{A}$ corresponds to stable $\mathrm{CW}$ out-of phase fields: the intensity of each laser reaches a steady state while the relative phase goes to $\pi$ so that the total intensity vanishes. Region B corresponds to a regime in which the electric field of each laser reaches a stationary pulsating regime in which both lasers emit intensity pulses synchronously. However, the relative phase goes to $\pi$, thus, while the intensity of each individual laser is self-pulsating and pulses are synchronous, the total intensity vanishes.

For $\varepsilon_{d}>0$ and $\varepsilon_{c}>0$, the OP supermode is always stable while the IP supermode is always unstable. For $\varepsilon_{d}<0$ and $\varepsilon_{c}<0$, the situation is reversed: the OP supermode is always unstable while the IP supermode is always stable. Thus, if $\varepsilon_{d}$ and $\varepsilon_{c}$ have the same sign, no chaotic behavior is found, and only coherent regimes are displayed.

\section{Chaotic Behavior}

Chaotic attractors arise in semiconductor lasers due to phase instabilities related to extra degrees of freedom, such as external injection [23], feedback [24], or mutual coupling as in the present case. However, the specific route to chaos depends on the specific structure. In our system, chaos originates from the interplay of the $\alpha$-factor and the imaginary part of the coupling $\varepsilon_{c}$. Indeed, when either $\alpha=0$ or $\varepsilon_{c}=0$, no chaotic behavior occours. Physically, the $\alpha$-factor reverts the intensity pulsations to phase pulsations, while $\varepsilon_{c}$ induces supplementary phase oscillations. In our system, there are two different chaotic attractors, depending on whether the absorber is saturated or not. Indeed, in the unstable regions $\mathrm{C}$ and $\mathrm{D}$ of Fig. 4, two different routes to chaos are found.

In region $C$ the two lasers are pulsating with an irregular amplitude and phase. When crossing from region $B$ to region $C$, the IP supermode has an instability coming from the relative phase subset (32)-(35). Therefore, the instability is in a direction transverse to the subspace embedding the intensity pulses. This leads to a regime where both lasers emit chaotic intensity pulses which are desynchronized. The total intensity reflects the sum of the two incoherent chaotic dynamics (see Fig. 7), showing large pulses separated by practically vanishing intensity.

When crossing from region A to region D, the IP supermode also has an instability coming from the relative phase subset (32)-(35). In this case, since no pulses were present in region $\mathrm{A}$, the emerging regime is characterized by large-amplitude excursions in the direction transverse to the intensity subspace followed by decaying oscillations over the stable subspace of the IP solution (see Fig. 8)

Regions $\mathrm{C}$ and $\mathrm{D}$ are separated by the Hopf-bifurcation locus shown as a horizontal solid line at $A_{p}=5.6$ in Fig. 4. Thus, intrapulse oscillations decay toward a fixed point in region $\mathrm{D}$, whereas they approach a limit cycle (the one generated by the Hopf bifurcation) in region C. Both scenarios (in regions $\mathrm{C}$ and D) lead to the emergence of a chaotic behavior with different attractors (see Fig. 9). Notice that, for the chaotic attractor of region $\mathrm{C}$, the total intensity shows large excursions departing 

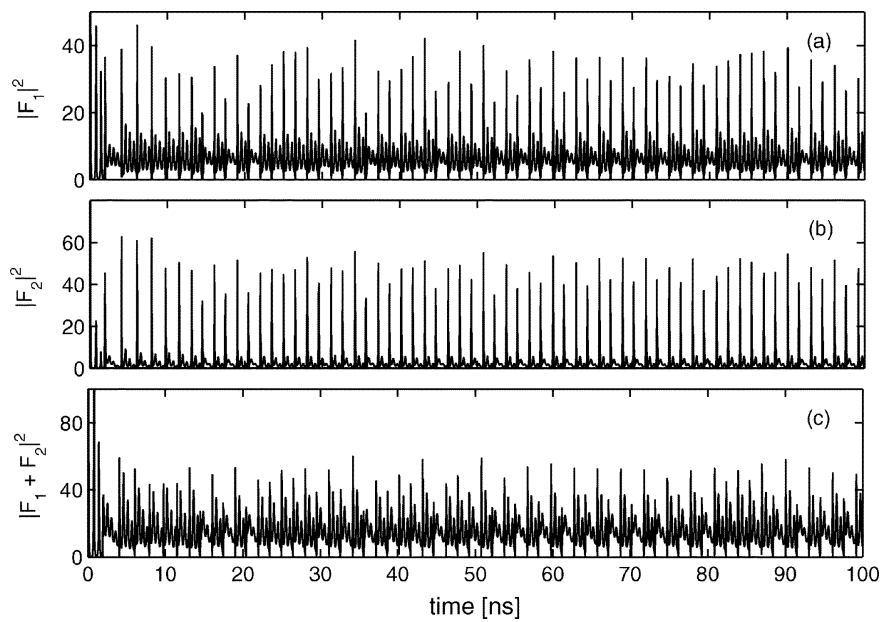

Fig. 8. Chaotic behavior with saturated absorber. $A_{p}=6, \varepsilon_{c}=0.4$, and $\varepsilon_{d}=-0.1$.
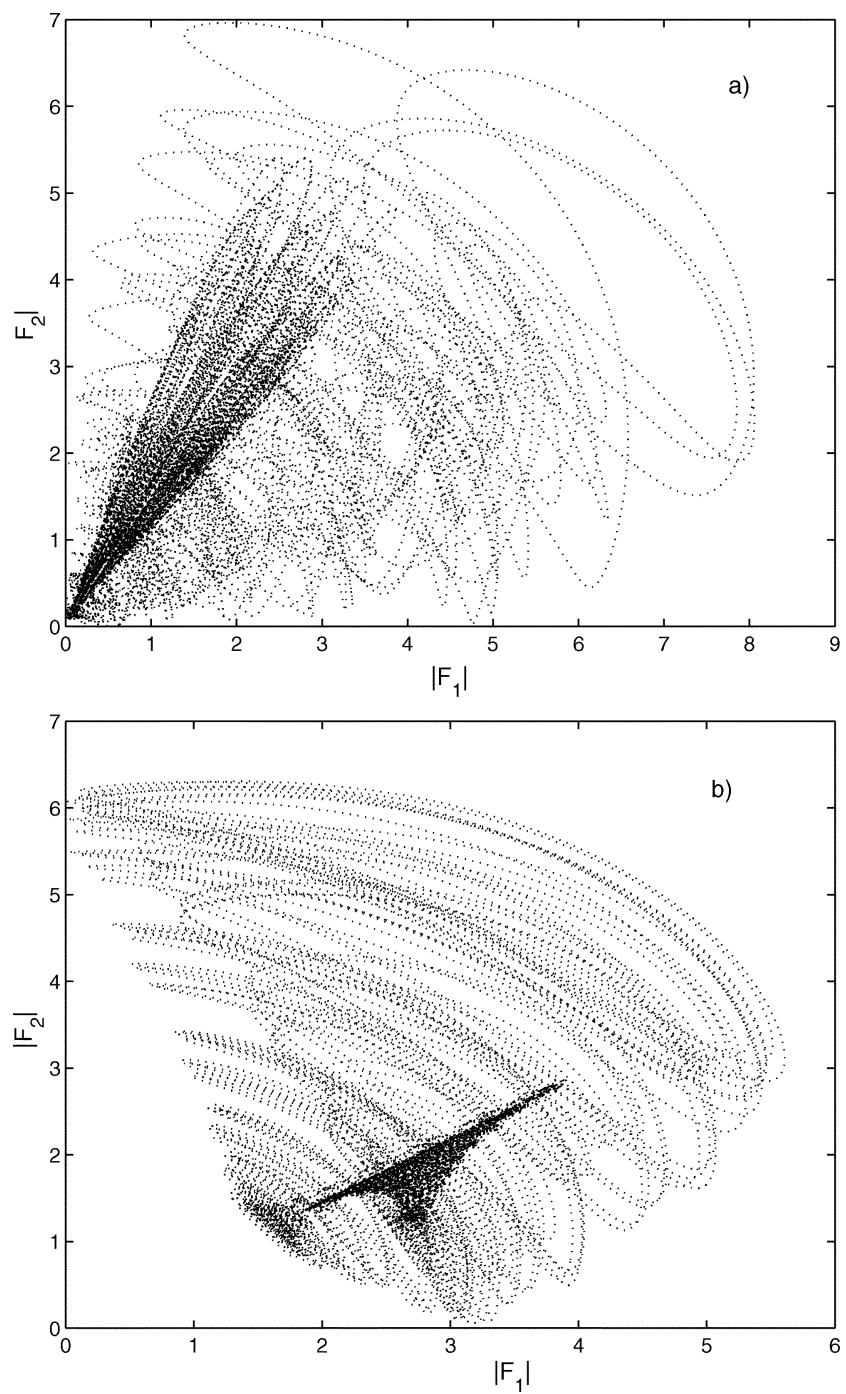

Fig. 9. (a) Map of the chaotic attractor with unsaturated absorber (region $\mathrm{C}$ of Fig. 4). The parameters of the system are $A_{p}=4$ and $\varepsilon_{c}=0.5$. (b) Map of the chaotic attractor with saturated absorber (region D in Fig. 4). $A=6$ and $\varepsilon_{c}=-0.1$.

from zero while in region $\mathrm{D}$ the total intensity never vanishes; on the contrary, excursions are departing from the previously stable stationary solution.

\section{SUMMARY}

We have introduced a model for the fields and carrier dynamics of two laterally coupled EELs, each containing an unpumped region acting as a saturable absorber. From analytical and numerical analysis, we demonstrated that coherent self-sustained pulsations with different relative phase relationships between the electric field in the two lasers are possible (self-pulsating in-phase or out-of-phase supermodes) for a wide range of parameters of the considered device. We have found two coherent regimes: stable $\mathrm{CW}$ in-phase and out-of-phase supermodes and in-phase and out-of-phase pulsating super-modes, where the the intensity of the superposition of the two fields is four times the intensity of the single field. This could represent a promising result in view of the possibility of synchronizing a many-element array of pulsating lasers. In addition, we have found and discussed two different routes to optical chaos.

\section{ACKNOWLEDGMENT}

The authors would like to thank F. d'Ovidio for the valuable insight on the nonlinear dynamics and G. Giuliani, M. Sorel, and J. Mulet for useful discussions and precious hints.

\section{REFERENCES}

[1] H. A. Haus, "Mode-locking of lasers," IEEE J. Select. Topics Quantum Electron., vol. 6, no. 6, pp. 1173-1185, Nov.-Dec. 2000.

[2] R. W. Dixon and W. B. Joyce, "A possible model for sustained oscillations (pulsations) in (Al, Ga)As double-heterostructure lasers," IEEE J. Quantum Electron., vol. QE-15, no. 6, pp. 470-474, Jun. 1979.

[3] T. L. Paoli, "Changes in the optical properties of cw (AlGa)As junction lasers during accelerated aging," IEEE J. Quantum Electron., vol. QE-13, no. 5, pp. 351-359, May 1977.

[4] P. Peterson, A. Gavrielides, M. P. Sharma, and T. Erneux, "Dynamics of passively $Q$-switched microchip lasers," IEEE J. Quantum Electron., vol. 35, no. 8, pp. 1247-1256, Aug. 1999.

[5] C. R. Mirasso, G. H. M. van Tartwijk, E. Hernández-García, D. Lenstra, S. Lynch, P. Landais, P. Phelan, J. O. Gorman, M. S. Miguel, and W. Elsasser, "Self-pulsating semiconductor lasers: Theory and experiment," IEEE J. Quantum Electron., vol. 35, no. 5, pp. 764-770, May 1999.

[6] M. Yamada, "A theoretical analysis of self-sustained pulsation phenomena in narrow-stripe semiconductor lasers," IEEE J. Quantum Electron., vol. 29, no. 5, pp. 1330-1336, May 1993.

[7] A. Scirè, J. Mulet, C. R. Mirasso, and M. S. Miguel, "Total intensity and polarization self-pulsations in vertical-cavity surface-emitting lasers," Opt. Lett., vol. 27, no. 11, pp. 912-915, 2002.

[8] H. Kawaguchi, Bistabilities and Nonlinearities in Laser Diodes. London, U.K.: Artech House, 1994.

[9] G. P. Agrawal and N. K. Dutta, Semiconductor Lasers, 2nd ed. New York: Van Nostrand, 1993.

[10] P. Rees, P. McEvoy, A. Valle, J. O. Gorman, S. Lynch, P. Landais, L. Pesquera, and J. Hegarty, "A theoretical analysis of optical clock extraction using a self-pulsationg laser diode," IEEE J. Quantum Electron., vol. 35, no. 2, pp. 221-227, Feb. 1999.

[11] C. Juang, T. M. Hwang, J. Juang, and L. Wen-Wei, "A synchronization scheme using self-pulsationg laser diodes in optical chaotic communications," IEEE J. Quantum Electron., vol. 36, no. 3, pp. 300-304, Mar. 2000.

[12] A. Scirè, J. Mulet, C. R. Mirasso, J. Danckaert, and M. S. Miguel, "Polarization message encoding through vectorial chaos synchronization in vertical-cavity surface-emitting lasers," Phys. Rev. Lett., vol. 90, pp. 113 901(1)-113 901(4), 2003.

[13] G. Carpintero, B. J. Roycroft, R. Lemos, and P. Acedo, "Considerations on the rate equation description of a twin stripe laser array," Proc. SPIE, vol. 4646, pp. 381-387, 2002.

[14] D. Baoxun, "A theory on stability of double-section semiconductor lasers," IEEE J. Quantum Electron., vol. 25, no. 5, pp. 847-849, May 1989. 
[15] A. Paiss and I. Hardy, "A coupled-mode analysis of twin-stripe index-guided lasers," IEEE J. Quantum Electron., vol. 25, no. 7, pp. 1609-1616, Jul. 1989.

[16] A. W. Snyder and J. D. Love, Optical Waveguide Theory. New York: Chapman \& Hall, 1983.

[17] J. Y. Wang, M. Cada, R. V. Dommelen, and T. Makino, "Dynamic characteristic of bistable laser diodes," IEEE J. Select. Topics Quantum Electron., vol. 3, no. 5, pp. 1271-1279, Oct. 1997.

[18] O. Hess and E. Scholl, "Eigenmodes of the dynamically coupled twin-stripe semiconductor laser," Phys. Rev. A, Gen. Phys., vol. 50, pp. 787-792, 1994

[19] P. Ru, P. K. Jacobsen, J. V. Moloney, and R. A. Indik, "Generalized coupled-mode model for the multistripe index-guided laser arrays," $J$. Opt. Soc. Amer. B, Opt. Phys., vol. 10, pp. 507-515, 1993.

[20] M. Mnkel, F. Kaiser, and O. Hess, "Stabilization of spatiotemporally chaotic semiconductor laser arrays by means of delayed optical feedback," Phys. Rev. E, Stat. Phys. Plasmas Fluids Relat. Interdiscip. Top., vol. 56, pp. 3868-3875, 1997.

[21] T. W. Carr and T. Erneux, "Dimensionless rate equations and simple conditions for self-pulsing in laser diodes," IEEE J. Quantum Electron., vol. 37, no. 9, pp. 1171-1177, Sep. 2001.

[22] J. L. A. Dubbeldam and B. Krauskopf, "Self-pulsations of lasers with saturable absorber: Dynamics and bifurcations," Opt. Commun., vol. 159, pp. 325-338, 1999.

[23] V. Annovazzi-Lodi, A. Scirè, M. Sorel, and S. Donati, "Dynamic behavior and locking of a semiconductor laser subject to external injection," IEEE J. Quantum Electron., vol. 34, no. 12, pp. 2350-2357, Dec. 1998.

[24] V. Annovazzi-Lodi, S. Merlo, M. Norgia, and A. Scirè, "Characterization of a chaotic telecommunication laser for different fiber cavity lengths," IEEE J. Quantum Electron., vol. 38, no. 9, pp. 1171-1177, Sep. 2002.

Alessandro Scirè (M'99) was born in Rimini, Italy, on June 26, 1971. He received the S.M and Ph.D. degrees in electronic engineering and the S.M. degree in physics from the University of Pavia, Pavia, Italy, in 1995, 1999, and 2003, respectively.

He held a Marie Curie Individual Fellowship from 2000 to 2002 at the Instituto Mediterráneo de Estudios Avanzados (IMEDEA), Universitat des Illes Balears, Palma de Mallorca, Spain, where he is presently working. His research interests include laser dynamics, stochastic processes in nonlinear systems, cooperative phenomena in coupled oscillators, and dynamic networks.

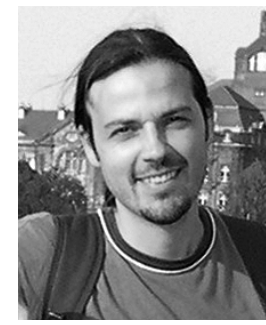

Claudio Juan Tessone was born in Cipolletti, Argentina, on March 15, 1974. He received the M.Sc degree in physics from the Instituto Balseiro, Universidad Nacional de Cuyo, Bariloche, Argentina, in 1999 . He is currently working toward the Ph.D. degree at the Instituto Mediterraneo de Estudios Avanzados (IMEDEA), Universitat des Illes Balears, Palma de Mallorca, Spain.

Since 2002, he has held a fellowship as Formacion del Personal Investigador of the Ministerio de Ciencia y Tecnología, Palma de Mallorca, Spain. His research interests are on interdisciplinary applications of physics to biology and other fields, including noise-induced phenomena (such as stochastic and coherence resonance), nonlinear systems, and synchronization of coupled nonlinear oscillators. He has coauthored ten journal papers.

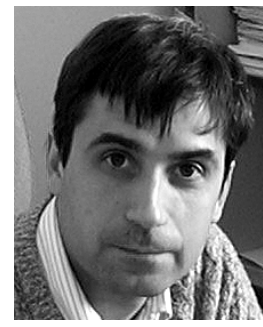

Pere Colet was born in Vilafranca del Penedés, Barcelona, Spain, on April 21, 1964. He received the M.Sc. degree from the Universitat de Barcelona, Barcelona, Spain, in 1987 and the Ph.D. degree from the Universitat de les Illes Balears, Palma de Mallorca, Spain, in 1991, both in physics.

In 1991, he became a Teaching Assistant with the Departament de Física, Universitat de les Illes Balears. From September 1991 to February 1993 and from April to September 1994, he was a Postdoctoral Fulbright Fellow with the School of Physics, Georgia Institute of Technology, Atlanta. In October 1994, he joined the Departament de Física, Universitat de les Illes Balears. Since May 1995, he has held a permanent research position with the Spanish Consejo Superior de Investigaciones Cientificas. He has coauthored 70 journal papers as well as 20 other scientific publications. His research interests include fluctuations and nonlinear dynamics of semiconductor lasers, synchronization of chaotic lasers and encoded communications, synchronization of coupled nonlinear oscillators, pattern formation and quantum fluctuations in nonlinear optical cavities, and dynamics of localized structures. 